\title{
Heat and hydrolytic enzymes treatment improved the Agrobacterium-mediated transformation of recalcitrant indica rice (Oryza sativa L.)
}

\begin{abstract}
Reported Agrobacterium transformation efficiency of indica rice shoot apices varied from 0.4 to $13.8 \%$. In order to improve the transformation efficiency, modification of transformation protocols through heat and hydrolytic enzyme treatments on rice shoot apices were carried out. Transient expression study using reporter genes revealed that shoot apices heat treated for $3 \mathrm{~min}$ at $42{ }^{\circ} \mathrm{C}$ during bacterial immersion showed improved GFP (63.0\%) and GUS (42.5\%) expressions per plant as compared to standard protocol (34.0\% GFP and $36.25 \%$ GUS). Shoot apices pre-treated with hydrolytic enzymes containing macerase, pectinase and cellulase at concentration ratio of 1:1:1 (w/v) also demonstrated high percentage of transient GFP (40.0 \%) and GUS (35.0 \%) expressions per plant. PCR analyses further confirmed the presence of GFP and GUS genes in the transformants. Stable expressions of GFP and GUS were also obtained in multiple shoots of regenerated shoot apices after 4 weeks of culturing in shoot proliferation media without hygromycin. In conclusion, the transformation efficiencies were improved significantly when heat (15.83\%) and hydrolytic enzymes (16.67 $\%$ ) were applied as individual treatments as compared to the standard transformation method which only accounted for $5.83 \%$.
\end{abstract}

Keyword: Agrobacterium; Heat; Hydrolytic enzymes; Rice; Shoot apices 